\title{
Heat-transfer analysis of microfabricated thermocapillary pumping and reaction devices
}

\author{
Timothy S Sammarco and Mark A Burns $\dagger$ \\ Department of Chemical Engineering, University of Michigan, Ann Arbor, MI 48109, USA
}

Received 4 March 1999, in final form 8 December 1999

\begin{abstract}
A heat-transfer analysis was performed on thermocapillary pumping, a surface-tension-based drop pumping mechanism. The analysis, which incorporates both fluid flow and energy transport through multiple device layers, reveals effective material, design, and operational choices that result in improved pumping performance. Important design factors include thermal conductivity, channel/substrate thickness, and the velocity of the liquid drops. Results for pumping a drop of water on a fused silica substrate with a glass channel show that uniform interface temperatures can be achieved with bottom heating as long as drop velocities remain below $\sim 0.1 \mathrm{~cm} \mathrm{~s}^{-1}$. The analysis was also extended to include thermal reaction channels. The reactor analysis verified that relatively uniform reaction temperatures were attainable with bottom heating as long as channel heights were below $\sim 100 \mu \mathrm{m}$.
\end{abstract}

\section{Introduction}

Recent developments have shown that microfabrication techniques offer a promising technology for miniaturizing and integrating chemical analysis systems (Burns et al 1996, 1998, Burke et al 1997, Cefa et al 1994, van der Berg and Bergvald 1995, Ramsey 1997, Anderson et al 1997, Marshall and Hodgson 1998). Potential applications include chemical process monitoring, medical diagnostics, and environmental testing. The increasing interest in microfabricated fluidic devices for use in chemical analysis has introduced new engineering challenges such as developing integrated, microfluidic, liquid-pumping systems.

Most proposed micro-pumps are mechanically based (i.e. contain moving parts) and are designed for moving continuous streams of liquids through microchannel systems. A discrete-volume pumping system, on the other hand, would minimize liquid usage, utilize well defined sample volumes, allow for independent sample control, and offer better sample containment. One such discrete drop system, called thermocapillary pumping (TCP), has been introduced as a mechanism for pumping discrete liquid samples within microfabricated liquid analysis devices (Burns et al 1996, Sammarco and Burns 1999). TCP is based upon the temperature dependence of surface tension; temperature changes induce changes in capillary pressure at the menisci of discrete liquid drops. The temperature-induced capillary pressure differences result in the recirculating (closedstreamline) flow of the drop. Consequently, the successful application of TCP is dependent upon the device's ability

$\dagger$ To whom correspondence should be addressed. to control the liquid temperatures at the menisci (air-liquid interfaces) of the drop.

In this paper we will present an analysis of the energy transport occurring in various designs for microfabricated TCP devices. The energy analysis includes transport effects from both heat conduction and liquid convection (closed-streamline flow), and the results are intended to identify effective design options for such systems. Other closed-streamline heat and fluid transfer analyses have been reported, including discrete drop flow in a tube (Duda and Vrentes 1971a, b, Oliver and Young Hoon 1968) and natural convection in rectangular cavities (Wilkes and Churchill 1966). These analyses concentrated on the behaviour of the liquid region given a set of imposed, and fixed, liquid boundary conditions. However, too often the conditions present in the liquid regions are dependent upon more global conditions as expressed through intermediate layers such as the channel and substrate walls. Consequently, we have extended our analysis to include both the regions surrounding the liquid drop and the external boundary conditions imposed (from the outside) upon these border regions. Our extended system includes the channel walls, heater substrates, air spaces, and ambient conditions surrounding the entire device. By including these surrounding regions, devices that heat rapidly, precisely, uniformly, and efficiently (i.e. low power) can be more accurately designed. Due to these additional system complexities, we opted not to pursue a rigorous twodimensional (2D) flow solution, but rather to incorporate a simpler one-dimensional (1D) laminar flow solution. Note that, although a 1D flow solution is used, a three-dimensional (3D) heat transfer system is being modelled (similar to a 1D flow solution describing a flow through a $3 \mathrm{D}$ pipe). 


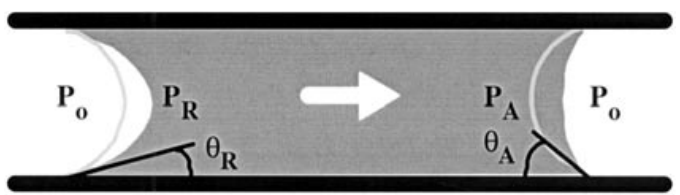

Figure 1. Discrete liquid drop showing curved menisci at the drop ends. The meniscus curvature produces a pressure difference between the liquid and air phases. By inducing a temperature change between the drop ends, a capillary pressure difference is created and causes drop motion. This surface tension-induced discrete drop movement is called TCP.

\section{Thermocapillary pumping (TCP)}

TCP is a non-mechanical, surface-tension driven pumping system for moving discrete liquid drops within microfabricated channels (Burns et al 1996, Sammarco and Burns 1999). Surface-tension effects at the ends of discrete liquid drops produce curved interfaces or menisci (figure 1). The meniscus curvature produces a pressure difference between the liquid and air region, called capillary pressure. This capillary pressure is described by the following form of the Young-Laplace equation (Sammarco and Burns 1999)

$$
P_{g}-P_{l}=\frac{G \sigma \cos \theta}{d} .
$$

In this equation $P_{g}$ is the interface pressure on the gaseous side, $P_{l}$ is the interface pressure on the liquid side, $\theta$ is the contact angle (see de Gennes 1985, Dussan 1979 for reviews) between the liquid and solid regions, $\sigma$ is the liquid surface tension, $d$ is the channel diameter (height), and $G$ is a constant specific to the channel geometry ( $G=4$ for circular, $G=2$ for slit-like, and $G \approx 2(1+$ height/width) for square and rectangular).

A change in the surface tension on one side of a liquid drop will induce a pressure difference across the liquid drop. This pressure difference induces bulk motion of the drop. By heating the receding interface of a single drop, the surface tension is reduced at that meniscus according to the following relationship (see Jasper 1972):

$$
\sigma=a-b T
$$

where $a$ and $b$ are positive empirical constants. The resulting TCP velocity, which is directed away from the heated zone, is based upon the Poiseuille flow model for flow through a slit (Rose and Heins 1962, Yarnold 1938, Richter et al 1997) and is given by (Sammarco and Burns 1999)

$$
v=\frac{d G b \cos \theta_{R}}{L S \mu}\left[\Delta T-\left(\frac{a}{b}-T_{A}\right)\left(1-\frac{\cos \theta_{A}}{\cos \theta_{R}}\right)\right]
$$

where

$$
\Delta T=T_{A}-T_{R}
$$

and $S$ is a channel shape constant, $\mu$ is liquid viscosity, $L$ is drop length, and $T_{A, R}$ is the interface temperature with the subscript $A$ referring to the advancing end of a moving drop and $R$ referring to the receding end. As evident in (3), the TCP velocity is driven by the induced temperature difference $(\Delta T)$ between the ends of a single liquid drop (Sammarco and

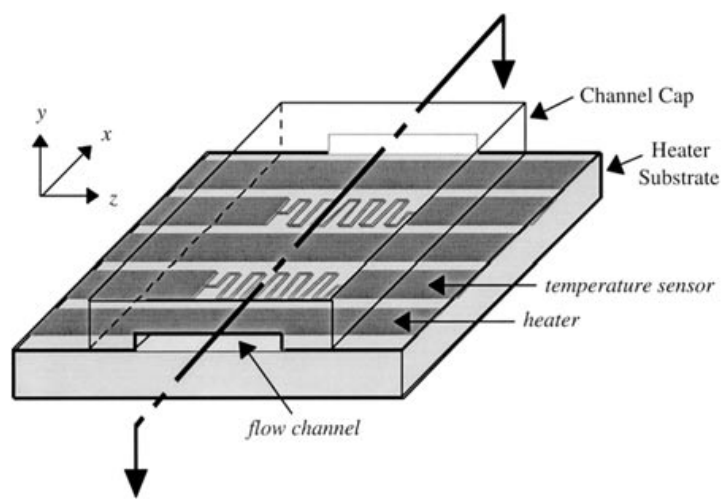

(a)

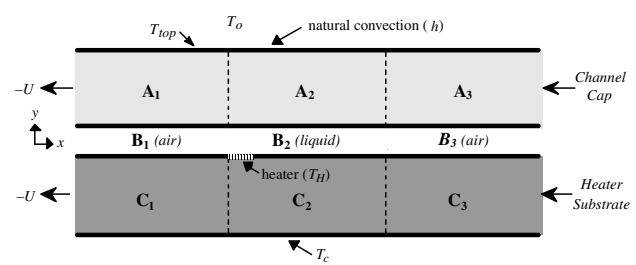

(b)

Figure 2. (a) 3D TCP device structure showing the flow channel, heaters, and temperature sensors. (b) $2 \mathrm{D}$ cross-section of a TCP device showing a discrete liquid drop sandwiched between the solid channel top cap and the lower heater substrate. The system is viewed in a moving reference frame in which the top and bottom layers are translating to the left at velocity $U$ (drop velocity); in this reference frame the average liquid velocity and the air space velocity are both zero.

Burns 1999). Consequently, precise temperature control is vital to the successful application of TCP as a drop movement mechanism for microfabricated devices.

Such precise temperature control is only possible if the devices used for TCP have been properly designed for the desired thermal performance. An effectively designed TCP system should be able to induce a uniform temperature at the heated interface. This desire is complicated by the difficulty in fabricating devices that can heat the interface from both the top and the bottom. Consequently, much of the analysis to follow will examine systems in which one-sided heating (from below only) occurs (see figure 2(a)). Another concern is that interface heating must remain relatively localized to the receding interface. If the temperature at the advancing meniscus rises significantly upon heating of the receding interface, the induced temperature-difference driving force for TCP will be diminished. Lastly, the heater power should be low for efficiency and to prevent thermal cross-talk.

\section{Mathematical model}

In the following model development, fluid flow and heat transfer are considered in two dimensions, $x$ and $y$, as shown in figure 2(b), with both $x$-axis directions extending indefinitely. Temperatures across the channel width are assumed to be uniform since the devices are heated by constant-temperature heater elements that extend across the entire width. The system is also considered at steady state with regard to both the temperature and the fluid flow because of the short characteristic times for establishing fully 
developed flow and steady-state temperatures at microscales. For modelling purposes, the drop's menisci will be flat and perpendicular to the channel surface (see figure 2(b)); the effect of this assumption should be insignificant due to the relatively small liquid volume in the curved portions of the interface. The entire system will be viewed in a reference frame moving with the drop to allow for a single heater to be permanently fixed beneath the receding drop interface. In practice, however, a series of heater elements would be sequentially activated along the flow channel bottom to follow the receding interface during TCP. Drop location detectors, such as a conductivity or capacitance sensor, could locate the receding menisci and allow for the proper heater sequence to move the drop (as proposed in Burns et al 1996, 1998). Again, in practice, the active heater would likely shift position slightly relative to the interface. The result may be a slightly diminished pumping efficiency when the heater is behind the interface and the $\Delta T$ driving force is lowered.

\subsection{Model equations}

The system being modelled is schematically represented in figure 2(b). The device, which consists of a top channel cap and a bottom heater substrate, is divided into nine sections $\left(A_{1}-A_{3}, B_{1}-B_{3}, C_{1}-C_{3}\right)$. The central rectangular region, $B_{2}$, represents the liquid drop undergoing $\mathrm{TCP}$-note that for modelling purposes the drop interfaces are flattened. Heating occurs at a small segment along the bottom corner of $\mathrm{B}_{2}$ at the receding interface. Associated with each of the nine regions comprising the device system is an energy equation and boundary conditions that can be solved together to obtain the temperatures throughout the entire device, including the liquid drop.

In TCP, energy transfer occurs via two mechanisms, heat conduction and fluid convection. Considering the 2D energy transfer in a circulating drop undergoing TCP with one velocity component (in the $x$-direction), we find that the energy equation (Bird et al 1960) reduces to

$$
\rho C_{p} v \frac{\partial T}{\partial x}=k\left(\frac{\partial^{2} T}{\partial x^{2}}+\frac{\partial^{2} T}{\partial y^{2}}\right)
$$

where $\rho$ is the liquid density, $C_{p}$ is the constant pressure heat capacity, $k$ is the thermal conductivity, $T$ is temperature, $x$ is the axial coordinate along the channel length, $y$ is the coordinate along the liquid interface perpendicular to $x$, and $v$ is the liquid velocity in the $x$-direction (which is a function of $y$ ). Note, a uniform temperature is assumed across the channel width since the heaters extend across the channel and are at a constant temperature.

The boundary conditions around the outer boundaries of the whole device are

channel top

$$
\left.k_{m} \frac{\partial T}{\partial y}\right|_{m}=h\left(T_{T o p}-T_{0}\right) \quad \text { (free convection) }
$$

substrate bottom

$$
T_{\text {bottom }}=T_{0} \quad \text { (constant temperature) }
$$

left, right ends

$$
\frac{\partial T}{\partial x}=0 \quad \text { (infinitely wide) }
$$

while the inner boundary conditions between the various regions within the device are

constant- $x$ interface

$$
\left(k \frac{\partial T}{\partial x}\right)_{m}=\left(k \frac{\partial T}{\partial x}\right)_{n} \quad \text { (conservation of energy) }
$$

constant- $y$ interface

$$
\left(k \frac{\partial T}{\partial y}\right)_{m}=\left(k \frac{\partial T}{\partial y}\right)_{n} \quad \text { (conservation of energy) }
$$

heater surface

$$
T_{\text {heater }}=T_{H} \quad \text { (constant temperature) }
$$

The following dimensionless variables

temperature:

$$
\bar{T}=\frac{T-T_{0}}{T_{H}-T_{0}}
$$

velocity

$$
\bar{v}=\frac{v}{U}
$$

$x$-coordinate

$$
\bar{x}=\frac{x}{L}
$$

$y$-coordinate

$$
\bar{y}=\frac{y}{d}
$$

are used to reduce equation (5) to a dimensionless equation. Consequently, the dimensionless energy equation for each of the nine regions of the device (see figure 2) becomes

$$
\left[(P e \lambda) \bar{v} \frac{\partial T}{\partial \bar{x}}=\left(\lambda^{2}\right) \frac{\partial^{2} \bar{T}}{\partial \bar{x}^{2}}+\frac{\partial^{2} \bar{T}}{\partial \bar{y}^{2}}\right]_{A 1-A 3, B 1-B 3, C 1-C 3} .
$$

The above system of nine identical equations contain the following dimensionless groups:

Peclet Number $(P e)=\frac{\text { rate of fluid convection }}{\text { rate of thermal diffusion }}$

$$
=\frac{\rho C_{p} \mathrm{~d} U}{k}=\frac{\mathrm{d} U}{\alpha}
$$

$\lambda=\frac{d}{L}$

$=$ channel height-to-length aspect ratio for each region.

In the expressions above, $T_{H}$ is the heater temperature, $T_{c}$ is the cooler temperature at the bottom of the device, $\alpha$ is the thermal diffusivity of the system, $T_{0}$ is the surrounding air temperature, and $U$ is the average liquid velocity. Note, in most instances it will be assumed that $T_{c}=T_{0}$.

Similarly, the dimensionless boundary conditions become

device top

$$
\left(\frac{\partial \bar{T}}{\partial \bar{y}}\right)_{n}=N u\left(\bar{T}_{\text {surface }}-\bar{T}_{0}\right)_{n}
$$


device bottom

$$
\bar{T}_{\text {bottom }}=0
$$

left, right ends

$$
\frac{\partial \bar{T}}{\partial \bar{x}}=0
$$

constant $x$ interface

$$
\left(\frac{\partial \bar{T}}{\partial \bar{x}}\right)_{m}=\psi_{m n}\left(\frac{\partial \bar{T}}{\partial \bar{x}}\right)_{n}
$$

constant- $y$ interface

$$
\left(\frac{\partial \bar{T}}{\partial \bar{y}}\right)_{m}=\eta_{m n}\left(\frac{\partial \bar{T}}{\partial \bar{y}}\right)_{n}
$$

heater surface

$$
\bar{T}_{\text {heater }}=1
$$

where $N u$ (Nusselt number), $\eta_{m n}, \psi_{m n}$, and $\kappa_{n m}$ are defined as

$N u=\frac{h_{m} d_{n}}{k_{n}}$

(thermal resistance ratio, conduction to convection)

$$
\kappa_{n, m}=\frac{d_{n, m}}{k_{n, m}}
$$

(thermal resistance conduction of a layer/region)

$$
\eta_{m n}\left(\frac{d_{m}}{k_{m}}\right) /\left(\frac{d_{n}}{k_{n}}\right)
$$

(ratio of thermal resistances of adjacent layers)

$$
\psi_{m n}=\left(\frac{L_{m}}{k_{m}}\right) /\left(\frac{L_{n}}{k_{n}}\right)
$$

(ratio of thermal resistances of adjacent regions).

The parameters $\eta_{n m}$ and $\psi_{m n}$ are measures of the relative heat transfer resistances of neighboring regions/layers (i.e. region/layer $m$ to region/layer $n$ ). When viewed from the reference frame of the drop moving at a bulk velocity $U$, all of the device regions are moving (i.e. have a non-zero Peclet number) except for the air spaces around the drop. These air spaces are assumed to move in a plug flow manner and, thus, have a zero velocity in this reference frame.

\subsection{Velocity field}

Since the closed-streamline flow of a long drop is mostly onedimensional, with the exception of the extreme ends of the drop (Duda and Vrentes 1971a,b), a 1D, slit-flow solution for the liquid velocity profile was used. This liquid velocity profile, which is given in a reference frame of moving with the drop at velocity $U$, is used except at the interface nodes on the drop,

$$
\bar{v}=\frac{1}{2}-6 \bar{y}^{2} .
$$

The above velocity profile is only a function of the channel position perpendicular to the flow direction, $\bar{y}$, which is typical of flow through a slit (see Bird et al 1960). Note that $\bar{y}=0$ is located along the central axis of the liquid layer (i.e. the centerline of the liquid layer shown in figure 3 ).

To ensure a circulation of convective energy at the drop ends, both $\bar{y}$ coordinates (at a given $\bar{x}$ ) were identified that

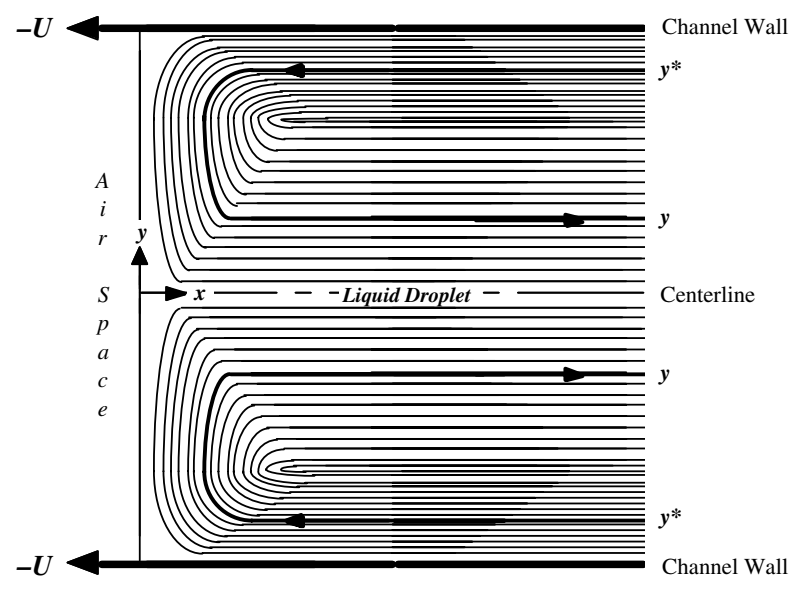

Figure 3. Circulating streamline patterns in the left interface of a discrete drop. The wall is translating at velocity $U$ to the left causing the streamlines near the wall to move to the left while the streamlines nearer the center of the drop move to the right. The interface is stationary in this reference frame although circulation occurs within the drop. Note $y$ and $y^{*}$ represent the vertical coordinates that are connected along the path of a single streamline as it circulates in both the top or bottom halves of the drop.

passed by a single, closed-streamline moving along $\bar{x}$. This is accomplished by choosing a $\bar{y}$ coordinate and integrating the velocity profile outward from the center of the circulation until a zero net volume was obtained, assuming constant density. The resulting expression relates the two $\bar{y}$-axis positions through which a single closed-streamline passes as it circulates on either the upper or lower halves of a moving liquid drop (see figure 3):

$$
\bar{y}^{*}=\frac{1}{2}\left(\frac{3}{2}-\bar{y}\right) \pm \sqrt{\left(\bar{y}-\frac{3}{2}\right)^{2}-4(\bar{y}-1)\left(\bar{y}-\frac{1}{2}\right)} .
$$

In equation (30), $\bar{y}^{*}$ is the corresponding $\bar{y}$-coordinate passed by a unique streamline that had previously passed through $\bar{y}$ (at the same $\bar{x}$ ) during the recirculating flow of a discrete drop.

\subsection{Temperature solution}

The energy equation in given as equation (16) is evaluated using the velocity field model given (equation (29)) and the explicit finite-difference expressions developed in the appendix. Once both $\bar{y}$-coordinates of a single streamline were identified for a given $\bar{x}$-coordinate, the convective portion of the finite-difference equation for 1D flow (equation (A1) in the appendix) was coupled to its corresponding streamline (determined from equation (30)). For instance, the first and second coefficients on the right-hand side of equation (A1) contains both conductive and convective portions,

$$
\begin{aligned}
& \frac{\lambda^{2}}{2\left(\lambda^{2}+\beta^{2}\right)} \pm \frac{\lambda \Delta \bar{x} \bar{u} P e}{2\left(\lambda^{2}+\beta^{2}\right)} \\
& =\text { conductive portion } \pm \text { convective portion }
\end{aligned}
$$

where the sign is plus (+) for the first coefficient and minus (-) for the second. The segregation of energy transport mechanisms coupled with the acceptance of (30) allows us to implement equation (A1) at the ends of a drop without solving for the exact flow solution. 
As an example, at the left end (advancing end) of a drop system such as shown in figure 2 , the end node at the interface is designated as $(i, j)$. By applying the above strategy one node in from the interface (i.e. at node $(i+1, j))$, we can associate the conductive portion of (31) with node $(i+1, j)$ while linking the convective portion with the flow into the node at $\left(i+1, j^{*}\right)$ (determined from (30)). At a distance from each interface equal to about one-half of a channel height, the streamlines become parallel as in Poiseuille flow (see Duda and Vrentes 1971a). This distance, $d / 2$, was chosen as the finite-difference step-size resulting in parallel flow at all interior nodal points; along the air-liquid interfaces a zero $x$-velocity is assumed. Consequently, the standard finitedifference expressions developed earlier are utilized at all but the air-liquid interfaces. At these interfaces, which are boundary points, the finite-difference equations developed for the corresponding boundary conditions are utilized.

\section{Results}

The combination of multiple material choices, stacked device layers (silicon, glass, quartz, polymers) and various packaging methods all result in numerous design options for microfabricating TCP devices. The thermal analysis results presented in this section are intended to guide in designing effective temperature controllable microfabricated TCP devices. Specifically, our objective is to identify TCP device designs that: achieve uniform meniscus temperatures at the heated interface, localize temperature increases to the heated interface, and require the lowest power outputs by the heaters. In the following sections we will examine how various factors influence these TCP-related heating objectives. The analysis will also be extended to temperaturecontrolled reaction channels where the entire substrate is heated beneath a static liquid drop in order to determine the uniformity of heating throughout the reaction mixture. Note that for presentation purposes in the results section, the $\bar{y}$-coordinate for the bottom surface of the liquid layer will be designated as $\bar{y}=0$ and the top surface as $\bar{y}=1$.

\subsection{Base-case device}

Figure 4 contains a 2D finite-difference result for TCP in a base-case device containing a $25 \mu \mathrm{m}$ high by $2.5 \mathrm{~mm}$ long water drop moving at $0.1 \mathrm{~cm} \mathrm{~s}^{-1}$. The water drop is located between a glass channel cap and a fused silica $\left(\mathrm{SiO}_{2}\right)$ substrate, each $500 \mu \mathrm{m}$ thick. Regions 1 and 3 in figure 2(b) were set at $40 \mathrm{~mm}$ long to ensure that the device behaves as if it extended indefinitely in both directions.

In this base-case device, the dimensionless temperature of the heated interface $(\bar{T})$ is not uniform, but varies by approximately $7 \%$ along the interface. This variation in $\bar{T}$ translates into only a $2{ }^{\circ} \mathrm{C}$ variation along the heated interface for a heater surface temperature of $50^{\circ} \mathrm{C}\left(T_{H}\right)$ and a device bottom temperature of $20^{\circ} \mathrm{C}\left(T_{c}\right)$. As we move axially away from the heater towards the non-heated end, the temperature decreases rapidly. At approximately one-third of the distance to the opposite interface, $\bar{T}$ decreases by about $90 \%$. In regard to TCP, this efficient axial cooling localizes the heating and effectively maximizes the temperature difference driving force for TCP (Sammarco and Burns 1999). Any heating of the opposite interface reduces the $\Delta T$ driving force and increases the average temperature of the drop. Obtaining localized heating during TCP may be necessary with some temperature-sensitive biological systems.

\subsection{Structural parameters (channel and substrate)}

Both the temperature profile and the power requirement of a TCP systems can be quite dependent on the characteristics of the channel and the substrate. Important characteristics associated with both the channel cap and the heater substrate include material properties, layer thickness, and boundary condition (upper and lower surfaces). Likewise, the thickness of the liquid layer also plays an important role in determining the temperature profile along the heated air-liquid interface.

In addition to the base-case device results obtained with glass channels (see figure 4), results were also obtained for devices containing silicon channels (500 $\mu \mathrm{m}$ thick) and plastic channels (10 $\mu \mathrm{m}$ thick, $p$-xylylene, also called Parylene-C; (Webster and Mastrangelo 1997)). In all three cases, $500 \mu \mathrm{m}$ thick fused silica $\left(\mathrm{SiO}_{2}\right)$ substrates were used. The plastic channels, which were only $10 \mu \mathrm{m}$ thick, yielded the most uniform temperatures along the heated drop interface with $\bar{T}$, changing by roughly $4 \%$ along the interface, as opposed to the $7 \%$ variation obtained with glass channels. The silicon channels (figure 5), however, result in a nearly $70 \%$ variation in $\bar{T}$ along the heated interface. This drastic change is due to the less resistant heat transfer properties of silicon (compared to glass and plastic, see table 1) relative to the liquid layer. Such a large temperature gradient along the interface may result in a non-uniform liquid interface pressure, leading to the selective flow of liquid down one side of a channel. Alternatively, these temperature gradients may induce secondary thermocapillary flows along the interface (Rashidnia and Balasubramaniam 1991). Although the consequence of such interfacial flows is uncertain, one possible effect is the formation of recirculation cells in the drop rather than a continuous, TCP-induced axial pressure difference.

In order to obtain uniform interface temperatures in the liquid layer, the lowest possible liquid-to-channel thermal resistance ratio is desired. This will ensure that the majority of the temperature decrease will occur in the channel layer; thus, the channel wall will act as an insulator for the liquid. In relation to the dimensionless groups presented earlier, it is desirable to have a liquid heat transfer resistance that is much lower than that of the channel wall. This ratio of liquid-to-channel heat transfer resistance is represented by the dimensionless group $\eta_{B_{2} A_{2}}$, which is contained in the boundary condition given by equation (23). Comparing $\eta_{B_{2} A_{2}}$ for all three channel materials $(0.12$ for glass, 0.35 for plastic, and 12 for silicon), we notice that both glass and plastic (Parylene-C) channels yield $\eta_{B_{2} A_{2}}$ values that are on the order of 0.1 while $\eta_{B_{2} A_{2}}$ is on the order of 10 for silicon. Based upon this observation, channels should be designed with $\eta_{B_{2} A_{2}}$ values of $\sim 0.1$ or smaller to achieve more uniform interface temperatures. Note, however, this is a generalization based upon a particular observation; several other factors can influence this result and need to be 


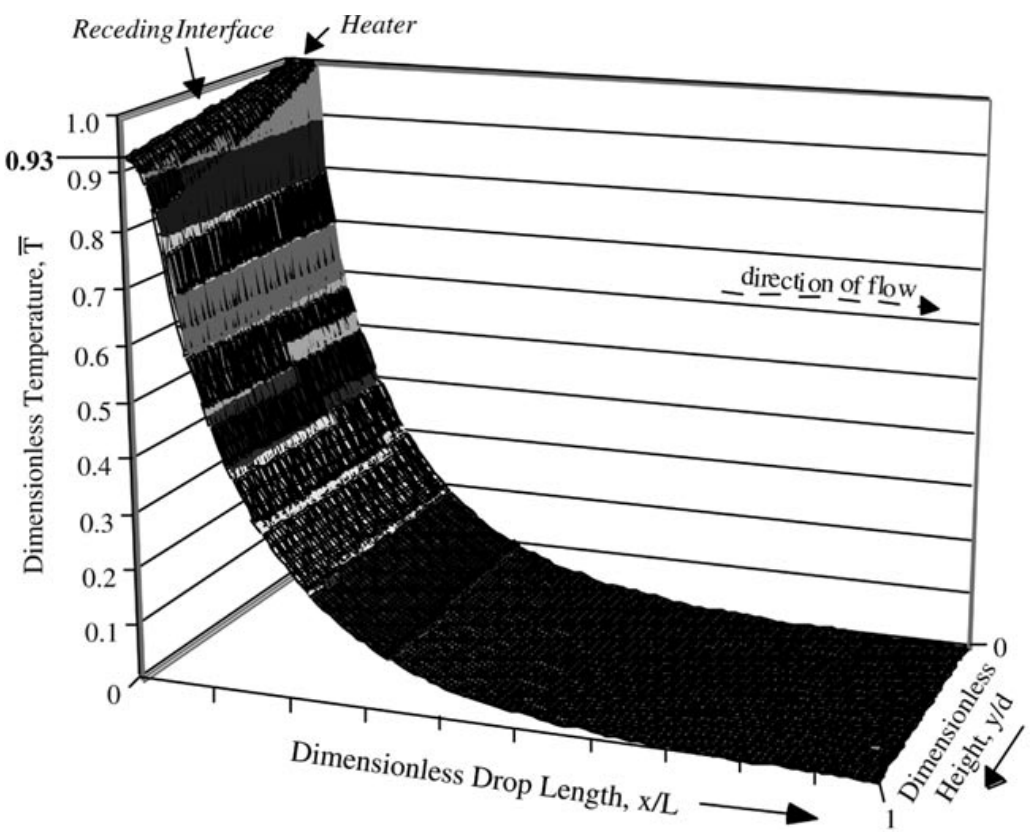

Figure 4. 2D dimensionless temperature distribution throughout the liquid phase of the base-case device-a water drop between a glass channel cap and a fused silica $\left(\mathrm{SiO}_{2}\right)$ heater substrate, both translating to the left at $U=0.1 \mathrm{~cm} \mathrm{~s}^{-1}$. The dimensionless temperature of the heated interface remains relatively uniform, varying by less than $7 \%$ along the interface. Also, the temperature increase at the heated interface remains localized to that interface. In this base-case device, a $500 \mu \mathrm{m}$ long heater is positioned at the interface with $400 \mu \mathrm{m}$ of heater length located in the air space and $100 \mu \mathrm{m}$ in the liquid space. $N u \sim 10^{-2}$ and $\eta_{B_{2} A_{2}} \approx 0.1$ for this case.

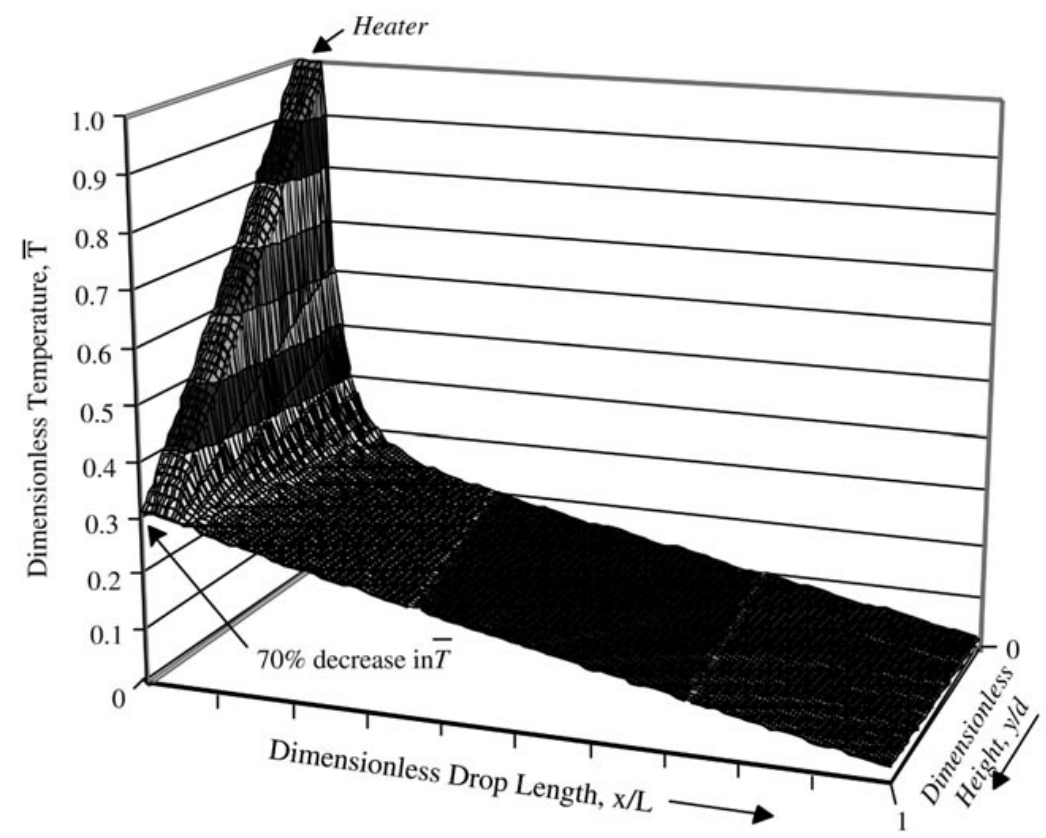

Figure 5. 2D dimensionless temperature distribution of the water layer in a variation of the base-case device in which the channel cap is constructed from silicon. The high thermal conductivity of silicon results in better heat transfer through the silicon cap relative to the liquid layer. The result is a $\sim 70 \%$ change in $\bar{T}$ along the heated interface. $N u \sim 10^{-4}$ and $\eta_{B_{2} A_{2}} \approx 12$ for this case.

considered. These additional factors include the boundary condition at the top surface of the channel (represented by the Nusselt number, $N u$ ), the liquid velocity $(U)$, the height of the flow channel $\left(d_{\text {channel }}\right)$, and the liquid itself. These issues will be addressed in later sections.

The power requirements for the three channel types were also examined but were shown to differ very little.
For instance, assuming that $T_{H}$ is $50^{\circ} \mathrm{C}$ and To is $20^{\circ} \mathrm{C}$, the required heater power output is roughly $4.7,4.9$, and $6.6 \mathrm{~W} \mathrm{~cm}^{-1}$ of heater width for glass, $p$-xylylene (Parylene$\mathrm{C}$ ), and silicon channels, respectively. On a per heater basis (500 $\mu \mathrm{m} \times 500 \mu \mathrm{m}$ heaters), the power requirements are 0.2 , 0.2 , and $0.3 \mathrm{~W} \mathrm{~cm}^{-1}$ for glass, $p$-xylylene (Parylene-C), and silicon, respectively. 


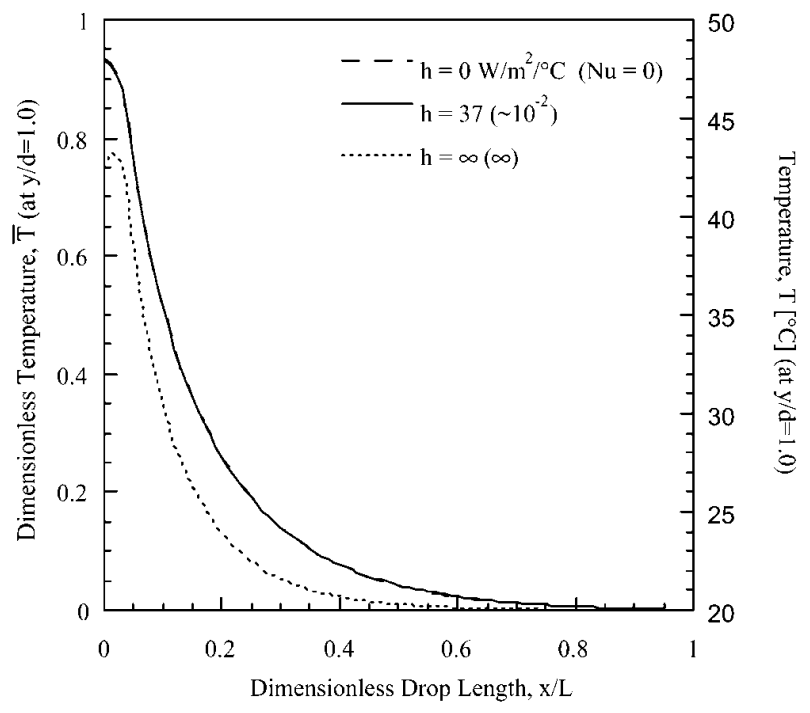

Figure 6. The effect of heat transfer coefficient, $h$, on the dimensionless axial temperature distribution along the top of a water drop $($ at $y / d=1)$ in the base-case device. Corresponding values for the dimensionless Nusselt number are also included for comparison (see equation (25)).

The results presented above utilized a free convection boundary condition based upon a heat transfer coefficient ( $h \approx 37 \mathrm{~W}{ }^{\circ} \mathrm{C}^{-1} \mathrm{~m}^{-2}$ ) calculated using a correlation for an upward facing, horizontal, flat plate (Chapman 1984). This upper boundary condition can also influence the thermal performance of TCP devices as shown by (19). This boundary condition is characterized by the Nusselt number, $N u$, which contains $h$ and is defined in equation (25). Figure 6 shows the results obtained for $h$ values of 0,37 , and $\infty \mathrm{W}{ }^{\circ} \mathrm{C}^{-1} \mathrm{~m}^{-2}$ in the base-case device. Results for the zero flux condition $(h=N u=0)$ are nearly identical to those obtained with the free convection boundary condition $\left(h=37 \mathrm{~W}{ }^{\circ} \mathrm{C}^{-1} \mathrm{~m}^{-2}\right.$,). As $h \rightarrow \infty(N u \rightarrow \infty$, i.e. $T_{\text {surface }}=T_{0}$ ), however, a nearly $25 \%$ change in $\bar{T}$ along the heated interface results. A cooling effect is also achieved in the remainder of the drop, as seen in figure 6.

As mentioned earlier, both $\eta_{B_{2} A_{2}}$ and $N u$ can influence the interfacial temperature profile at the heated end of a drop in the TCP system shown in figure 2. It is desirable to design systems with both $\eta_{B_{2} A_{2}}$ and $N u$ values as low as practical. In the examples presented above, acceptable values for $\eta_{B_{2} A_{2}}$ appear to be on the order of 0.1 or smaller while $h$ values on the order of forty or smaller seem reasonable. Unfortunately, these two dimensionless parameters are in opposition to attempts at reducing their sizes together. For instance, reducing $\eta_{B_{2} A_{2}}$ requires a decrease in $k_{\text {channel }}$ or an increase in $d_{\text {channel }}$ while reducing $N u$ requires an increase in $k_{\text {channel }}$ or a decrease in $d_{\text {channel }}$. This situation can be dealt with most simply by insulating the top channel surface, thereby, forcing $N u$ toward zero without affecting $\eta_{B_{2} A_{2}}$.

In addition to $\eta_{B_{2} A_{2}}$ being affected by the upper channel cap, the parameter also depends on the liquid layer thickness (i.e. flow channel height). Figure 7 shows the interface temperature profile in the base-case design (water) for different flow channel heights $\left(d_{\text {liquid }}\right)$. Relative to the $7 \%$ variation in $\bar{T}$ obtained with the base case thickness of $25 \mu \mathrm{m}$

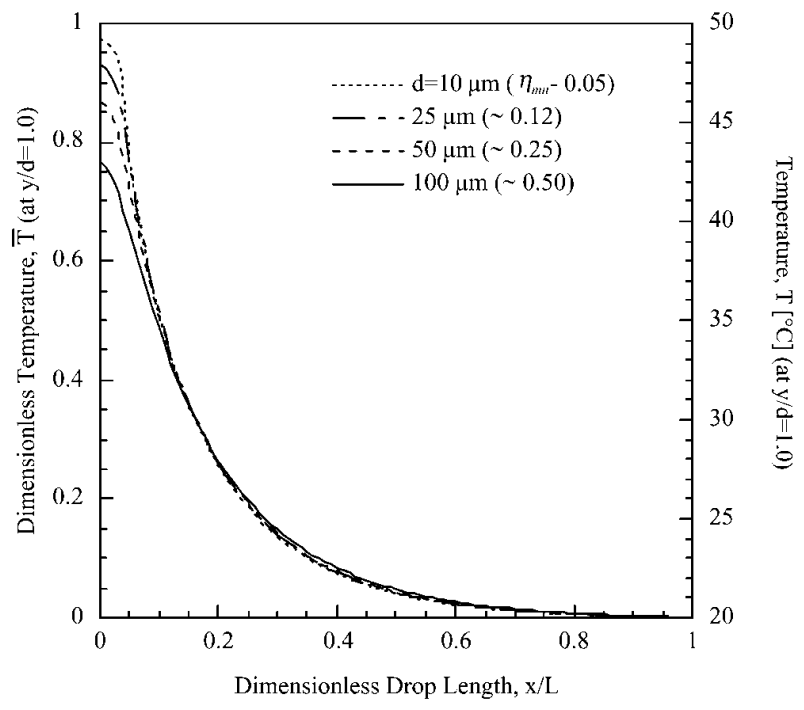

Figure 7. Effect of flow channel height $\left(d_{\text {liquid }}\right)$ on the dimensionless temperature profile along the top of a water drop in a base-case device with $U=0.1 \mathrm{~cm} \mathrm{~s}^{-1}$. As an example, a temperature scale corresponding to $T_{H}=50^{\circ} \mathrm{C}$ and $T_{c}=20^{\circ} \mathrm{C}$ was also included on the right axis.

$\left(\eta_{B_{2} A_{2}} \approx 0.1\right)$, a $10 \mu \mathrm{m}$ thick layer $\left(\eta_{B_{2} A_{2}} \approx 0.05\right)$ gives a more uniform interface temperature (3\% variation in $\bar{T}$ ) while a $50 \mu \mathrm{m}$ thick layer $\left(\eta_{B_{2} A_{2}} \approx 0.2\right)$ gives a larger variation in $\bar{T}(13 \%)$. This result is consistent with basic heat-transfer behaviour through two slabs in series. As the thickness of the liquid layer decreases, the relative heat transfer resistance of the channel wall to the liquid layer increases (i.e. $\eta_{B_{2} A_{2}}$ decreases). A small $\eta_{B_{2} A_{2}}$ is desired for more uniform air-liquid interface temperatures. Based upon the examples examined earlier, $\eta_{B_{2} A_{2}}$ on the order of 0.1 and smaller should be sought. As an extreme example, a $100 \mu \mathrm{m}$ fluid layer $\left(\eta_{B_{2} A_{2}} \approx 0.5\right)$ yields a $23 \%$ variation in $\bar{T}$ along the heated interface. If such thicker fluid layers are desired, for instance $100 \mu \mathrm{m}$, then $\eta_{B_{2} A_{2}}$ should be decreased either by increasing the channel wall thickness or by using a material with a lower thermal conductivity (see table 1). Changes to the thickness of the liquid layer also affect both the liquid layer's Peclet number $(\mathrm{Pe})$ and ratio of drop height/length $(\lambda)$, which influences the drop velocity (see Sammarco and Burns 1999). The influence of the Peclet number $(P e)$ in the liquid layer will be addressed in a later section.

The final structural component to be examined is the heater substrate. Variations in substrate materials were also compared for their affect on the temperature uniformity of $\bar{T}$ at the heated interface. Materials chosen for the heater substrate comparison include silicon, fused silica $\left(\mathrm{SiO}_{2}\right)$, and polycarbonate (a polymer, Webster et al 1998). Finitedifference results for these three substrates (each $500 \mu \mathrm{m}$ thick) show that there is only a slightly difference in $\bar{T}$ along the heated interface between these three cases. For example, a $7 \%$ change in $\bar{T}$ was obtained for $\mathrm{SiO}_{2}$ and silicon at $y / d=1$, while a $10 \%$ variation was obtained with polycarbonate substrates. The largest variation in results between the different substrates (silicon, $\mathrm{SiO}_{2}$, polycarbonate) was in the heater power output of each case. Figure 8 shows the power output per unit channel width $\left(\mathrm{W} \mathrm{cm}^{-1}\right)$ necessary to obtain 


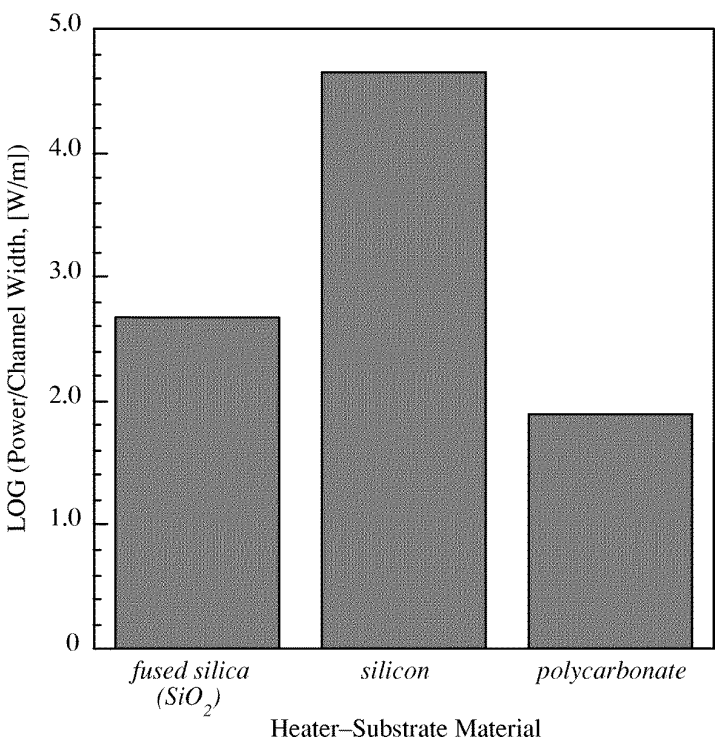

Figure 8. Comparison of power output requirements for fused silica $\left(500 \mu \mathrm{m}\right.$ thick $\left.\mathrm{SiO}_{2}\right)$, silicon $(500 \mu \mathrm{m})$, and plastic polycarbonate $(500 \mu \mathrm{m})$ heater substrates. As was in the case with silicon channel caps, the high thermal conductivity of silicon allows for enhanced heat transfer into the heater substrate rather than to the liquid layer.

a heater temperature of $50{ }^{\circ} \mathrm{C}$ for all three designs (given a $20^{\circ} \mathrm{C}$ ambient temperature). At $455 \mathrm{~W} \mathrm{~cm}^{-1}$, the power requirement for silicon is about two orders of magnitude higher than for either $\mathrm{SiO}_{2}$ or polycarbonate substrates. Whether this effect on power requirement becomes a practical issue, however, depends upon the limits imposed by a given system. If the power usage and heat dissipation are not an issue, all three materials could be used. From an economic perspective, however, polymer substrates may offer the least expensive option as long as they can withstand the operating temperatures needed for the application.

\subsection{Operational parameters (velocity, liquid, and heater location)}

The remaining factors influencing the thermal performance of TCP devices are referred to as operational parameters since they are determined by how a device is operated. These parameters include the magnitude of the pumping velocity $(U)$, the choice of liquid, and the location of the active heater in relation to the drop interface.

Figure 9 shows the effect of the average drop velocity on both the axial and interface temperature distributions for a water drop in the base-case TCP device. These results indicate that there is little difference between the pure conduction $\left(U=0 \mathrm{~cm} \mathrm{~s}^{-1}\right)$ results and those obtained for velocities below about $0.01 \mathrm{~cm} \mathrm{~s}^{-1}$. The optimal velocity appears to be roughly $0.1 \mathrm{~cm} \mathrm{~s}^{-1}$ (base-case device). At this velocity $\bar{T}$ varies by less than $10 \%$ along the heated interface while the temperatures in the remainder of the drop are lower than in cases with velocities of $\sim 0.01 \mathrm{~cm} \mathrm{~s}^{-1}$ and smaller. As the velocity increases to $1 \mathrm{~cm} \mathrm{~s}^{-1}$, however, convective heat transfer begins to dominate resulting in a larger temperature gradient along the heated interface.

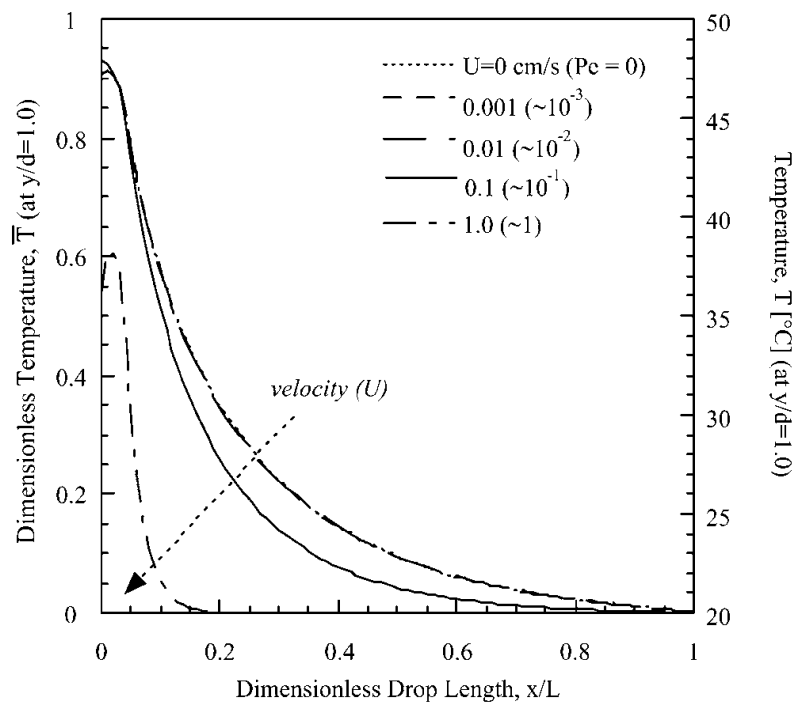

Figure 9. The effect of channel wall velocity $(U)$ on the dimensionless temperature profile at $y / d=1$ for a drop of water in the base-case device. At velocities below $\sim 0.001 \mathrm{~cm} \mathrm{~s}^{-1}$, the convective loses do not change the temperature distribution, but at velocities approaching $\sim 1 \mathrm{~cm} \mathrm{~s}^{-1}$, substantial interface temperature gradients exist. For comparison, a temperature scale in degrees centigrade was added to the right axis for a system where $T_{H}=50^{\circ} \mathrm{C}$ and $T_{0}=20^{\circ} \mathrm{C}$.

In terms of dimensionless quantities, liquid velocity $U$ enters the energy transport equation (equation (16)) through the Peclet number $(\mathrm{Pe})$, which is defined by (17). The higher the Peclet number, the more influence fluid convection, relative to conduction, has on defining the temperature profiles in the liquid layer. In the examples above, conduction appears to dominate energy transport when the Peclet number is $\sim 10^{-2}\left(\sim 0.01 \mathrm{~cm} \mathrm{~s}^{-1}\right.$ or lower). The result is that all such cases behave very similarly to the pure conduction case. When the velocity increases to the apparent optimal Peclet number of $\sim 10^{-1}\left(\sim 0.1 \mathrm{~cm} \mathrm{~s}^{-1}\right)$, a beneficial balance apparently occurs between conduction and convection. At this value, it appears that convection is significant enough to reduce the overall average temperature of the drop, thus better isolating the heating location without significantly disturbing the interface temperatures. As the Peclet number increases to $\sim 1\left(1 \mathrm{~cm} \mathrm{~s}^{-1}\right)$, fluid convection dominates energy transport resulting in a lower overall average liquid temperature but also in a large interfacial temperature gradient. Such large gradients are undesirable in TCP since a uniform capillary pressure is desired across the interface. Note that, in addition to be driven by an induced temperature difference, TCP velocity also depends on the liquid drop's viscosity, surface tension, and contact angles (Sammarco and Burns 1999).

Large liquid drop velocities can be compensated for by reducing the thickness of the liquid layer $(d)$ and thus reducing the Peclet number (see equation (17)). Unlike the velocity, decreasing $d_{\text {liquid }}$ improves the interface temperature uniformity by reducing the relative heat transfer resistance of the liquid layer, $\eta_{B_{2} A_{2}}$. In the velocity examples discussed above involving the base-case device, $\eta_{B_{2} A_{2}} \approx 0.1$. Alternatively, the channel height $(d)$ may also be used to adjust the Peclet number to compensate for unfavourable properties of the liquid $\left(k, C_{p}, \rho\right)$. Figure 10 shows the 


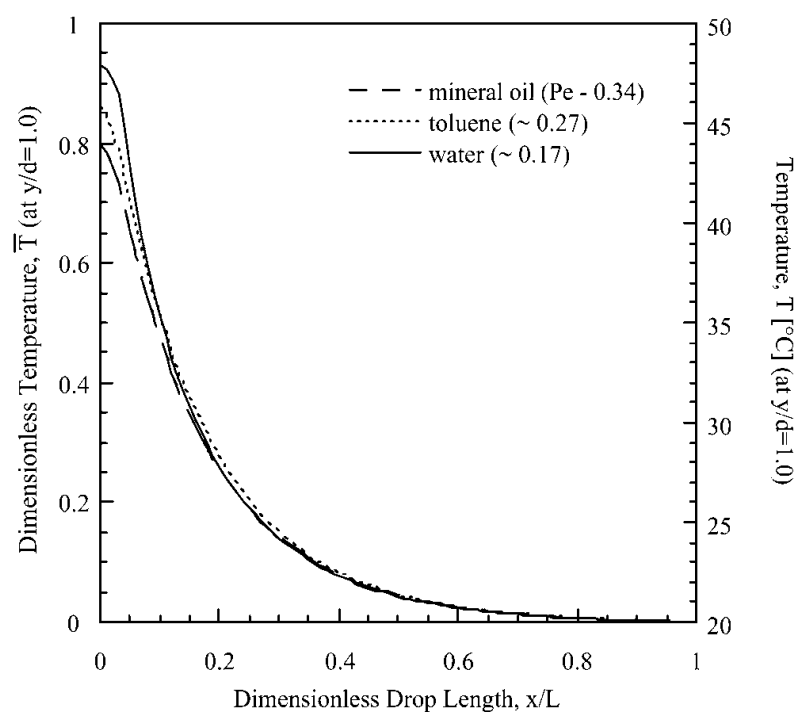

Figure 10. The effect of various liquids on the dimensionless temperatures along the top of a drop (i.e. at $y / d=1$ ) in the base-case device with a wall velocity of $0.1 \mathrm{~cm} \mathrm{~s}^{-1}$. For comparison, a temperature scale with $T_{H}=50^{\circ} \mathrm{C}$ and $T_{0}=20^{\circ} \mathrm{C}$ was included on the right axis.

effect of different liquids (see table 1) on the temperature distributions throughout the liquid region. As can be seen in figure 10 , the results for all three liquids indicate that water yields the most uniform interface temperatures.

The effect of drop velocity on heater power requirements is shown in figure 11 . The power requirement remains relatively constant for the drop velocities of $0.1 \mathrm{~cm} \mathrm{~s}^{-1}$ and lower. If the drop velocity increases to $1 \mathrm{~cm} \mathrm{~s}^{-1}$, the power requirements increase from $\sim 5 \mathrm{~W} \mathrm{~cm}^{-1}$ to $\sim 9 \mathrm{~W} \mathrm{~cm}^{-1}$ (assuming $T_{H}=50^{\circ} \mathrm{C}$ and $T_{0}=20^{\circ} \mathrm{C}$ ). Such an increase is due to the enhanced distribution of energy by enhanced fluid convection in the flow channel. Consequently, the heater must output more energy in order to maintain its surface temperature $\left(T_{H}\right)$ of $50{ }^{\circ} \mathrm{C}$. Note that the unit ' $\mathrm{W} \mathrm{cm} \mathrm{cm}^{-1}$, translates to watts per centimeter of heater width for heaters that are $500 \mu \mathrm{m}$ long along the channel length.

The last operational parameter to be discussed involves the location of the heater relative to the air-liquid interface. Since the heated area is actually a boundary between a liquid and a gas region, the location of the heater relative to this interface is itself an operational variable. Options for heater location include placing the heater beneath the air region, the liquid region, or split between both the air and liquid regions. Figure 12 shows the axial temperature profiles for these three cases. The different appearance of the temperature curves near the interface is due to the shifting relative heater position.

The design in which the heater was split equally between the air and liquid regions appears to be the most effective of the three. This case yields the highest average interface temperature while producing a lower average overall liquid temperature compared to the 'all liquid' case. The 'all air' example, in which the heater was entirely in the air space (100 $\mu \mathrm{m}$ away from the interface), yielded the lowest average overall liquid temperature. This design also produced a large variation in $\bar{T}$ along the heated interface, changing

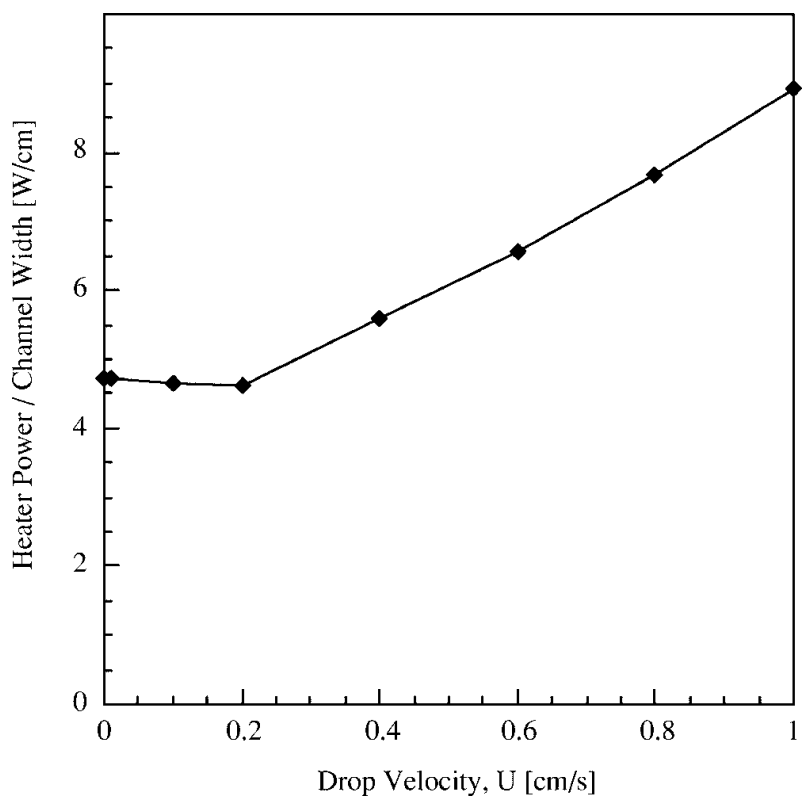

Figure 11. The effect of drop velocity on the heater power output (per unit channel width) required to maintain a fixed heater surface temperature $\left(T_{H}=50^{\circ} \mathrm{C}, T_{0}=20^{\circ} \mathrm{C}\right)$ assuming $500 \mu \mathrm{m}$ long heaters. The power reaches a minimum at a velocity of approximately $0.2 \mathrm{~cm} \mathrm{~s}^{-1}$ before increasing linearly with velocity as a result of enhanced convective energy loses.

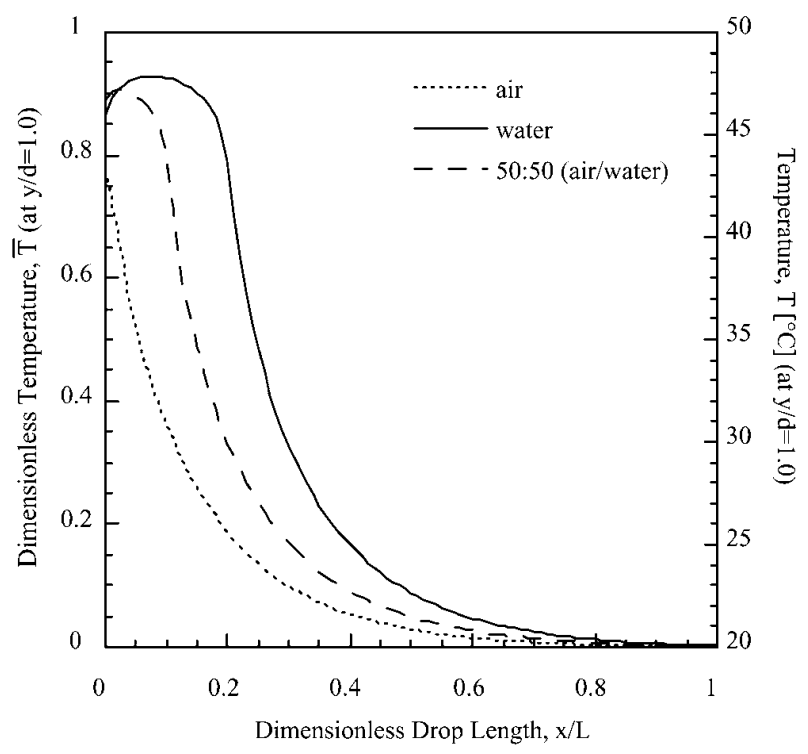

Figure 12. Dimensionless temperature distribution as it is affected by the relative location of the heater element with respect to the receding interface. Three heater locations are shown: entirely in the air space behind the interface ('air'), entirely within the liquid phase at the interface of the water drop ('water'), and split equally between the air and liquid phases crossing the interface ('50:50'). For comparison, a temperature scale in degrees centigrade was added to the right axis for a system where $T_{H}=50^{\circ} \mathrm{C}$ and $T_{0}=20^{\circ} \mathrm{C}$.

by $\sim 23 \%$. Although these three examples did not yield as uniform an interface temperatures as obtained in the standard 80:20 (air:liquid) ratio used for all of the previously discussed examples, they do demonstrate the importance of relative heater position in determining interface temperatures. 


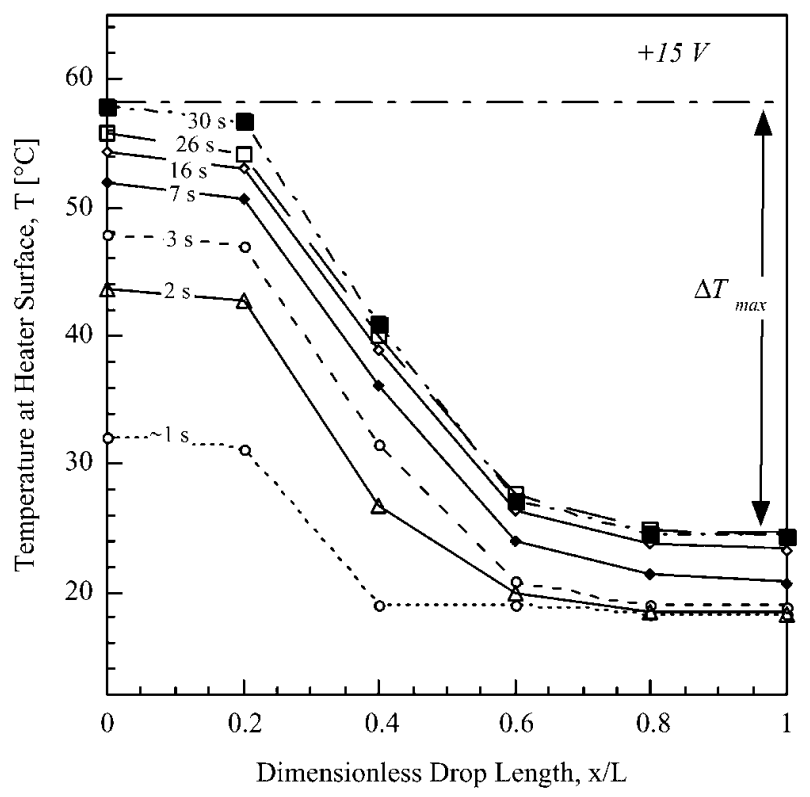

Figure 13. Transient experimental axial temperature distributions were measured through a drop of mineral oil using a microfabricated device similar to the base-case system described above. The temperature difference driving force is shown as the temperature difference between the extreme ends (interfaces) of the discrete drop, i.e. $\Delta T=T_{x / L=0}-T_{x / L=1}$. The temperature measurements were taken along the bottom of the liquid, i.e. at $y / d=0$. Steady-state conditions were approached after approximately $30 \mathrm{~s}$.

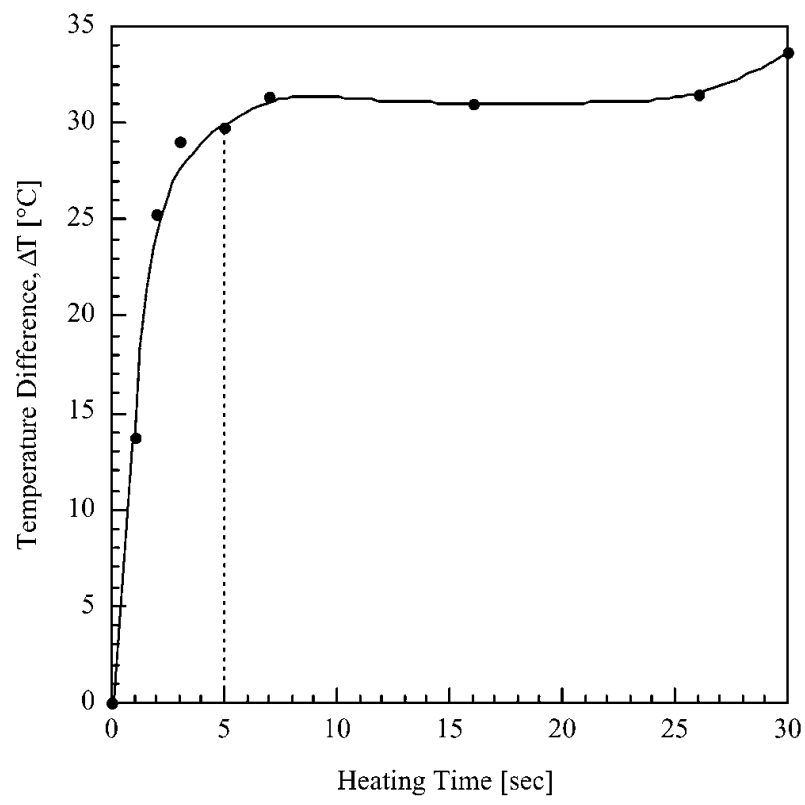

Figure 14. Transient temperature difference, $\Delta T$, data extracted from figure 14 . The temperature difference, $\Delta T$, reaches a steady-state value in approximately $5 \mathrm{~s}$. Interestingly, as shown in figure 13 , the individuals localized temperatures continue changing after $30 \mathrm{~s}$ of heating.

There were also noticeable differences in the power requirements between the three heater location designs. The power requirements for these designs are roughly 10, 25, and $45 \mathrm{~W} \mathrm{~cm}^{-1}$ for the 'all air', 'split equally', and 'all liquid' heater locations, respectively. These results were

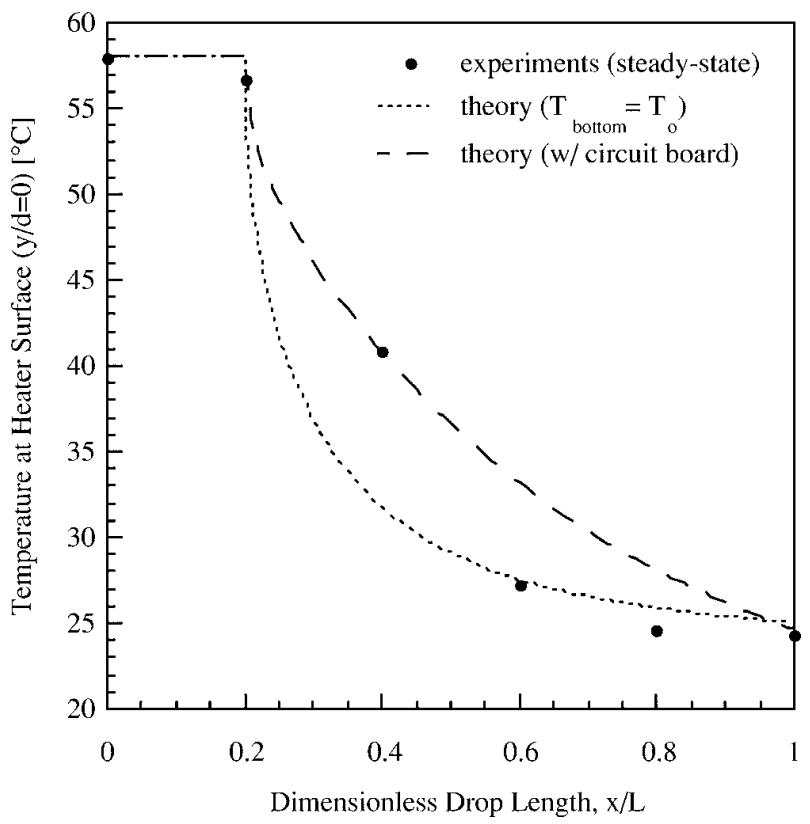

Figure 15. Comparison of the model predictions to the steady-state axial temperature data from figure 13 (full circles). Predictions were made using both a constant temperature, $T_{0}$, boundary condition beneath the heater substrate (dotted line) and a $2 \mathrm{~mm}$ thick circuit board beneath the heater substrate (dashed line). In the predictions, $T_{0}=25^{\circ} \mathrm{C}$ (room temperature), $T_{H}=58^{\circ} \mathrm{C}$, and $T_{c} \approx 0{ }^{\circ} \mathrm{C}(\bar{c}=-0.75)$ to account for active cooling beneath the circuit board.

obtained using the base-case device; however, the trend should translate to other designs. The relative heater location does not influence the dimensionless groups discussed earlier, but rather is simply a change in position of the constanttemperature boundary condition for the heater.

\subsection{Comparison to experiments}

To compare modelling results with their experimental counterparts, the base-case device discussed earlier was microfabricated as described by Sammarco and Burns (1999). This TCP system consists of a drop of mineral oil in a $\sim 2 \mathrm{~cm}$ long device constructed with a fused silica heater substrate and a glass channel cap, each $500 \mu \mathrm{m}$ thick. The bottom of the $25 \mu \mathrm{m}$ liquid flow channel was lined with $500 \mu \mathrm{m} \times 500 \mu \mathrm{m}$ planar heaters. Figure 13 shows the transient temperature distribution measured using resistive temperature detectors (RTDs) (see Sammarco and Burns 1999) throughout a single drop of mineral oil that is heated from beneath the meniscus at one end. After approximately $30 \mathrm{~s}$, the system begins to reach a steady-state temperature throughout the liquid. This long transience period is due to the relatively large length and thickness of a packaged microfabricated device compared to the size of a single heater element ( $250 \mu \mathrm{m}$ to $500 \mu \mathrm{m}$ long by $0.5 \mu \mathrm{m}$ thick). With respect to TCP, however, a pseudo steady state is achieved with regards to the temperature difference, $\Delta T$, between the drop ends. This temperature difference is the driving force for TCP and attains a steady value after approximately $5 \mathrm{~s}$ as shown in figure 14. This rapid attainment of a constant 


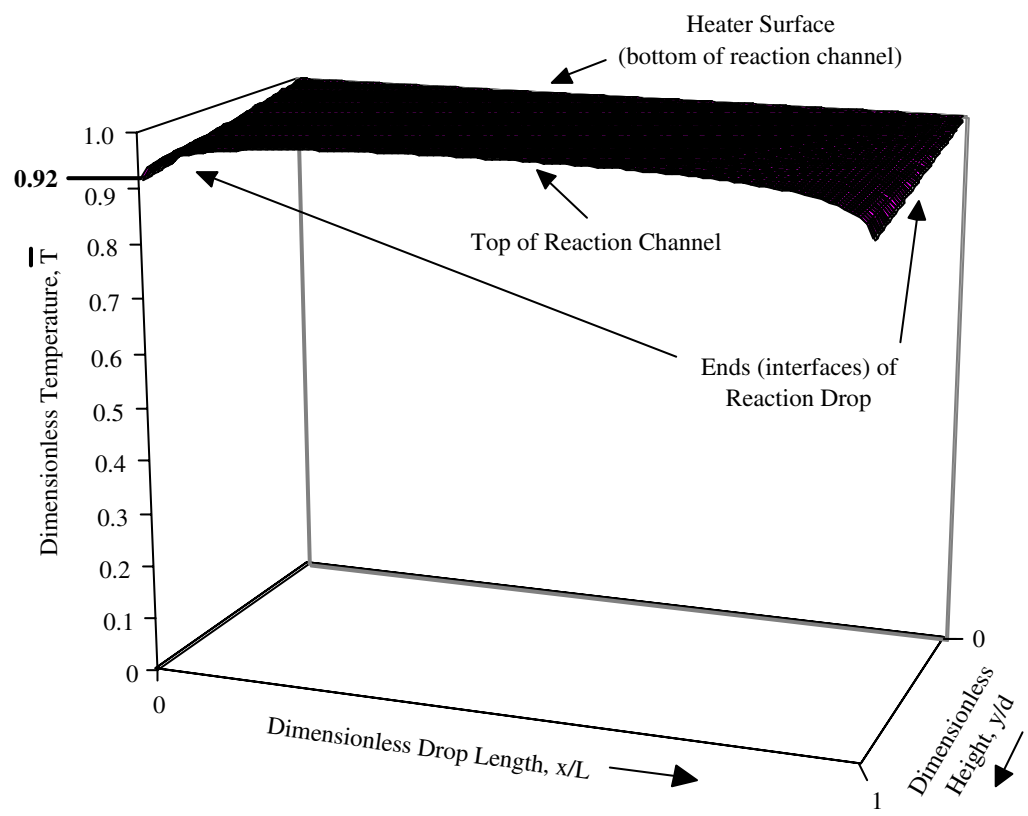

Figure 16. 2D dimensionless temperature distribution throughout a water drop in a $25 \mu \mathrm{m}$ high reaction channel (based on the base-case device). In this reactor example, the entire bottom surface of the reaction channel is heated. Notice that the liquid temperature remains very uniform except at the far drop ends. This example demonstrates the ability of bottom-sided heating to attain uniform reaction temperatures throughout the liquid reactant phase in small diameter reaction channels.

temperature difference, $\Delta T$, appears to confirm the steadystate assumption in the temperature model.

A comparison of modelling and experimental temperature results is shown for mineral oil in figure 15 . The experimental results show a more gradual temperature decay in a direction away from the heater than the standard model $\left(T_{\text {bottom }}=T_{0}\right)$ suggests. Both the experimental and standard model results do, however, show a leveling of temperature at the non-heated interface. When a $2 \mathrm{~mm}$ thick paper-phenolic circuit board (with a linear temperature distribution through it) is added beneath the heater substrate, the model predicts a slow and steady axial temperature decay without a leveling at the non-heated interface. The experimental results appear to fall somewhere in between these two model solutions.

Note that the circuit board is part of the device packaging; the packaging can vary significantly depending upon the application needs. These packaging options present numerous variations in conditions and materials that may be present beneath the microfabricated device. Consequently, the model results presented earlier implemented a constanttemperature boundary condition for the bottom of the device rather than assuming a specific packaging scheme. Based upon the results given in figure 15, these model results appear to fit the experimental data fairly well.

\subsection{Microfabricated reactors}

Another extremely important microfluidic component requiring precise thermal control is a micro-chemical reactor. Temperature control is required for many chemical analysis reactions such as the enzymatic reactions performed in DNA analysis protocols (Burns et al 1996, 1998, Burke et al 1997). A microreactor, in its simplest form, can consist of a microfluidic channel with heaters and temperature detectors beneath the channel for active temperature control. The energy equation given by (16) can be reduced for reactors since the liquid is typically stationary during a reaction, i.e. $U=0$. Consequently, (16) reduces to,

$$
\left[\lambda^{2}\left(\frac{\partial^{2} \bar{T}}{\partial \bar{x}^{2}}\right)+\frac{\partial^{2} \bar{T}}{\partial y^{2}}=0\right]_{A 1-A 3, B 1-B 3, C 1-C 3}
$$

and applies to each of the nine regions represented in figure 2(b).

Figure 16 shows the $2 \mathrm{D}$ temperature profile in a $25 \mu \mathrm{m}$ high reactor channel that is heated across the entire bottom surface of the liquid layer. Although heating occurs on the bottom side only, a near uniform temperature is attained throughout the liquid drop. These results suggest that onesided heating designs are sufficient for attaining uniform temperatures in small diameter flow channels. As the diameter of the channel increases, a larger temperature gradient is produced as shown in figures 16 and 17 . In instances when single-sided heating is not sufficient, such as in the $250 \mu \mathrm{m}$ high flow channel example, a dual heater design can be implemented (i.e. heaters are added to both the upper and lower surfaces of the flow channel). Also contained in figures 16 and 17 is a temperature profile produced by a dual-sided (top and bottom) heater design in a $250 \mu \mathrm{m}$ high reactor channel. The dual heater design yields a uniform liquid temperature throughout the entire drop, thereby significantly improving the temperature control capabilities of large volume reaction channels.

\section{Summary}

Solutions to finite-difference models were obtained for the energy equation as applied to a single drop of liquid 


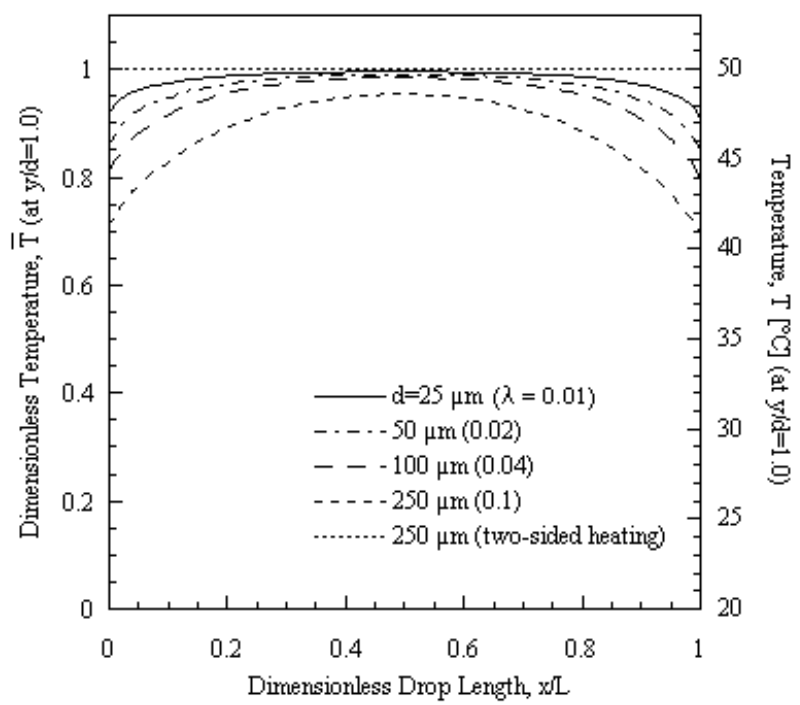

Figure 17. Dimensionless temperatures predicted for a micro-reactor channel being heated from the bottom side only. In the low profile channels, a near uniform temperature is achieved throughout the liquid phase (water) simply by heating from the bottom. As the height of the channel increases to $250 \mu \mathrm{m}$, a second heater is needed along the top of the liquid in order to obtain a uniform temperature throughout the liquid drop. For comparison, a centigrade temperature scale was added to the right axis for a system where $T_{H}=50^{\circ} \mathrm{C}$ and $T_{0}=20^{\circ} \mathrm{C}$.

undergoing TCP within a multilayer, micro-fabricated device. Results of the analysis show that, with single-sided heating, near uniform interface temperatures can be obtained in devices with $\mathrm{SiO}_{2}$ or plastic channel caps as opposed to those made from silicon. Designs with silicon channel caps result in significant temperature gradients along the interface due to the high thermal conductivity of silicon relative to other materials such as glass, fused silica, and plastic. The use of silicon as a substrate material, however, yields near uniform interface temperatures, but at the expense of much higher power requirements for the heater. These material effects associated with the channel cap were quantified using the dimensionless parameter $\eta_{B_{2} A_{2}}$, which is a relative measure of the heat transfer resistances of adjacent layers. Based upon model results, devices designs should aim for a $\eta_{B_{2} A_{2}}$ value of $\sim 0.1$ or less in order to obtain interface temperatures that vary by only a few degrees centigrade.

In addition to material effects, other factors such as pumping velocity, heater location in relation to the air-liquid interface, layer thickness, and pumping liquid all influence the temperature uniformity along the heated interface. Velocities up to $\sim 0.1 \mathrm{~cm} \mathrm{~s}^{-1}$ appear to behave similar to cases of pure conduction; however, significant temperature gradients are produced as the velocity increases to $\sim 1 \mathrm{~cm} \mathrm{~s}^{-1}$ due to the enhanced convective heat transfer effects. A comparison of Peclet numbers for the various velocity cases revealed that a $P e \sim 0.1$ or smaller should be sought in order to achieve more uniform interface temperatures.

The results of the analysis presented above are intended to guide the designs of microfluidic systems that integrate electronic temperature control components. The usefulness of these results has been extended to the analysis of microreaction systems. As with TCP devices, microreactors benefit from the same thermal property advantages associated with the micro-scales and small thermal masses of these devices. These characteristics make it possible to design devices that can be heated rapidly, precisely, uniformly, and efficiently (i.e. with low power). The results demonstrate the remarkable capability of microfabricated devices to achieve desired thermal performance when designed properly.

\section{Appendix. Finite-difference solution}

Equation (16) was evaluated using the explicit finitedifference equations that were obtained after substituting the derivative terms with their corresponding central difference approximations (for example see Carnahan 1969):

$$
\begin{aligned}
\bar{T}_{i, j} & =\left(\frac{\lambda^{2}-\Delta \bar{x} \lambda \bar{u} P e}{2 \lambda^{2}+2 \beta^{2}}\right) \bar{T}_{i+1, j}+\left(\frac{\lambda^{2}+\Delta \bar{x} \lambda \bar{u} P e}{2 \lambda^{2}+2 \beta^{2}}\right) \bar{T}_{i-1, j} \\
& +\left(\frac{\beta^{2}}{2 \lambda^{2}+2 \beta^{2}}\right)\left[\bar{T}_{i, j+1}+\bar{T}_{i, j-1}\right]
\end{aligned}
$$

where

$$
\beta=\frac{\Delta \bar{x}}{\Delta \bar{y}}
$$

the subscript $i$ refers to nodal increments along the $x$-coordinate, and the subscript- $j$ refers to increments along the $y$-coordinate. The above finite-difference expression is valid for the non-boundary nodes in all nine regions of the device shown in figure 2 . Notice, the unknown temperature at node $(i, j)$ is a function of the temperatures of the four neighboring nodes.

To implement the boundary conditions given earlier, the derivative terms in (22) and (23) must be evaluated for each region. For instance, consider the fluid (air or liquid)-solid (channel or substrate) boundaries of our TCP system in figure 2 . The boundary condition governing this interface is given by (23) where region- $m$ represents the fluid region and region- $n$ represents the solid region. The derivatives of temperature with respect to the $x$-direction can be replaced by their central difference counterparts to determine the flux of energy across a boundary in the $y$-direction. Since each energy equation is applicable to a particular region, however, the central difference approximation for the secondorder $y$-derivative cannot be used since it contains terms from the adjacent region. Consequently, to evaluate this boundary condition, the first-order partial derivative of temperature, with respect to $y$, is evaluated for each region by performing a Taylor expansion into region- $m$ around the interface temperature. Doing so yields an approximation for the second-order derivative at the interface of region- $m$ (for example see Carnahan 1969):

$$
\left.\frac{\partial^{2} \bar{T}}{\partial \bar{y}^{2}}\right|_{i, j} \approx \frac{2}{(\Delta \bar{y})^{2}}\left[\bar{T}_{i, j+1}-\bar{T}_{i, j}-\Delta \bar{y}\left(\frac{\partial \bar{T}}{\partial \bar{y}}\right)_{i, j}\right] .
$$

An expansion into region- $n$ yields the a similar approximation:

$$
\left.\frac{\partial^{2} \bar{T}}{\partial \bar{y}^{2}}\right|_{i, j} \approx \frac{2}{(\Delta \bar{y})^{2}}\left[\bar{T}_{i, j-1}-\bar{T}_{i, j}+\Delta \bar{y}\left(\frac{\partial \bar{T}}{\partial \bar{y}}\right)_{i, j}\right] .
$$

Substituting equations (A3) and (A4) into (16) and rearranging yields the following approximation for the 
first-order derivative in the boundary condition given by equation (23),

$$
\begin{aligned}
& \left(\left.\frac{\partial \bar{T}}{\partial \bar{y}}\right|_{i, j}\right)_{m} \approx\left(\frac{\lambda^{2}}{2 \beta^{2} \Delta \bar{y}}+\frac{\lambda \bar{u} P e}{4 \beta}\right)_{m} \bar{T}_{i+1, j} \\
& \quad+\left(\frac{\lambda^{2}}{2 \beta^{2} \Delta \bar{y}}-\frac{\lambda \bar{u} P e}{4 \beta}\right)_{m} \bar{T}_{i-1, j}+\left(\frac{1}{\Delta \bar{y}}\right)_{m} \bar{T}_{i, j+1} \\
& \quad-\left(\frac{\lambda^{2}}{\beta^{2} \Delta \bar{y}}+\frac{1}{\Delta \bar{y}}\right)_{m} \bar{T}_{i, j} .
\end{aligned}
$$

Similarly for region- $n$ we find,

$$
\begin{aligned}
& \left(\left.\frac{\partial \bar{T}}{\partial \bar{y}}\right|_{i, j}\right)_{n} \approx\left(\frac{\lambda^{2}}{2 \beta^{2} \Delta \bar{y}}-\frac{\lambda \bar{u} P e}{4 \beta}\right)_{n} \bar{T}_{i+1, j} \\
& \quad-\left(\frac{\lambda^{2}}{2 \beta^{2} \Delta \bar{y}}+\frac{\lambda \bar{u} P e}{4 \beta}\right)_{n} \bar{T}_{i-1, j}-\left(\frac{1}{\Delta \bar{y}}\right)_{n} \bar{T}_{i, j+1} \\
& \quad+\left(\frac{\lambda^{2}}{\beta^{2} \Delta \bar{y}}+\frac{1}{\Delta \bar{y}}\right)_{n} \bar{T}_{i, j}
\end{aligned}
$$

where the subscripts $m$ and $n$ refer to the region in which the parameters are evaluated.

Substitution of (A5) into (16) yields the following finite-difference formula for the interface temperature along boundaries of constant- $y$,

$$
\bar{T}_{i, j}=A \bar{T}_{i+1, j}+B \bar{T}_{i-1, j}+C \bar{T}_{i, j+1}+D \bar{T}_{i, j-1}
$$

where:

$$
\begin{aligned}
A & =\frac{\left(a_{m}-b_{m}\right)+\eta_{m n}\left(a_{n}-b_{n}\right)}{\left(2 a_{m}+c_{m}\right)+\eta_{m n}\left(2 a_{n}+c_{n}\right)} \\
B & =\frac{\left(a_{m}+b_{m}\right)+\eta_{m n}\left(a_{n}+b_{n}\right)}{\left(2 a_{m}+c_{m}\right)+\eta_{m n}\left(2 a_{n}+c_{n}\right)} \\
C & =\frac{c_{m}}{\left(2 a_{m}+c_{m}\right)+\eta_{m n}\left(2 a_{n}+c_{n}\right)} \\
D & =\frac{c_{n} \eta_{n m}}{\left(2 a_{m}+c_{m}\right)+\eta_{m n}\left(2 a_{n}+c_{n}\right)}
\end{aligned}
$$

and

$$
\begin{aligned}
a_{m, n} & =\left(\frac{\lambda^{2}}{2 \beta^{2} \Delta \bar{y}}\right)_{m, n} \\
b_{m, n} & =\left(\frac{\lambda \bar{u} P e}{4 \beta}\right)_{m, n} \\
c_{m, n} & =\left(\frac{1}{\Delta \bar{y}}\right)_{m, n} .
\end{aligned}
$$

For heat transfer in the direction of fluid motion (i.e. along boundaries of constant- $x$ ), a similar analysis yields the following finite difference expression for interface temperatures,

$$
\bar{T}_{i, j}=A^{\prime} \bar{T}_{i, j+1}+B^{\prime} \bar{T}_{i, j-1}+D^{\prime} \bar{T}_{i+1, j}+C^{\prime} \bar{T}_{i-1, j}
$$

where $A^{\prime}, B^{\prime}, C^{\prime}$, and $D^{\prime}$ are given by equations (A8)-(A11), but with $\eta_{m n}$ replaced by $\psi_{m n}$ and $a^{\prime}, b^{\prime}, c^{\prime}$ given as

$$
\begin{aligned}
a_{m, n}^{\prime} & =\left(\frac{\beta^{2}}{2 \lambda^{2} \Delta \bar{x}}\right)_{m, n} \\
b_{m, n}^{\prime} & =\left(\frac{\beta \bar{u} P e}{4 \lambda^{2}}\right)_{m, n}
\end{aligned}
$$

$$
c_{m, n}^{\prime}\left(\frac{1}{\Delta \bar{x}}\right)_{m, n} .
$$

At the top of the device, substitution of (A3) into (19) yields the following expression for the temperatures along the top of the device (assuming a constant average heat transfer coefficient, $h$, along the top of the device),

$$
\begin{aligned}
\bar{T}_{i, j} & =\left(\frac{a_{n}-b_{n}}{2 a_{n}+c_{n}+N u}\right) \bar{T}_{i+1, j}+\left(\frac{a_{n}+b_{n}}{2 a_{n}+c_{n}+N u}\right) \bar{T}_{i-1, j} \\
& +\left(\frac{N u}{2 a_{n}+c_{n}+N u}\right) \bar{T}_{i, j+1}+\left(\frac{c_{n}}{2 a_{n}+c_{n}+N u}\right) \bar{T}_{i, j-1}
\end{aligned}
$$

where $a_{n}, b_{n}$, and $c_{n}$ are given by (A12)-(A14) and are evaluated for region- $n$, the solid region from which heat transfer occurs to the surrounding environment (region- $m$ ).

\section{References}

Anderson R C, Bogdan G J, Barniv Z, Dawes T D, Winkler J and Roy K 1997 Microfluidic biochemical analysis systems Transducers '97 1997 Int. Conf. on Solid-State Sensors and Actuators vol 2, p 477

Bird R B, Stewart W E and Lightfoot E N 1960 Transport Phenomena (New York: Wiley) pp 46, 319

Burke D T, Burns M A and Mastrangelo C 1997 Microfabrication technologies for integrating nucleic acid analysis Genome Res. 7189

Burns M A, Mastrangelo C H, Sammarco T S, Man F P, Webster J R, Johnson B N, Foerster B, Jones D, Fields Y, Kaiser A R and Burke D T 1996 Microfabricated structures for integrated DNA analysis Proc. Natl Acad. Sci. USA 93 5556

Burns M A et al 1998 An integrated nanoliter DNA analysis device Science 282484

Carnahan B 1969 Applied Numerical Methods ed B Carnahan, $\mathrm{H}$ A Luther and J O Wilkes (New York: Wiley)

Cefa J J, Barrow D A, Woias P and Muller E 1994 Integrated chemical analysis microsystems in space life sciences research J. Micromech. Microeng. 4172

Chapman A J 1984 Heat Transfer (New York: Macmillan) pp 302-22

de Gennes P G 1985 Wetting: statics and dynamics Rev. Mod. Phys. 57827

Duda J L and Vrentas J S 1971a Heat transfer in a cylindrical cavity J. Fluid Mech. 45247

_ 1971b Heat transfer in a cylindrical cavity J. Fluid Mech. 45 261

Dussan E B 1979 The spreading of liquids on solid surfaces: static and dynamic contact angles Ann. Rev. Fluid Mech. 11371

Jasper J J 1972 The surface tension of pure liquid compounds J. Phys. Chem. Ref. Data. 1841

Marshall A and Hodgson J 1998 DNA chips: an array of possibilities Nature Biotechnol. 1627

Oliver D R and Young Hoon A 1968 Two-region non-Newtonian flow, part II: heat transfer Trans. Instn. Chem. Engrs. 46 T116

Ramsey G 1997 DNA chips: state of the art Nature Biotechnol. 16 40

Rashidnia N and Balasubramaniam R 1991 Thermocapillary migration of liquid droplets in a temperature gradient in a density matched system Exp. Fluids 11167

Richter M, Woias P and Weiß D 1997 Microchannel for applications in liquid dosing and flow rate measurement Sensors Actuators A 62480

Rose W and Heins R W 1962 Moving interfaces and contact angle rate dependency J. Colloid Sci. 1739

Sammarco T S and Burns M A 1999 Thermocapillary pumping of discrete drops in microfabricated analysis devices AIChE J. February 1999, p 350 
van der Berg A and Bergvald P 1995 Micro total analysis systems Proc. $\mu$ TAS'94 Workshop (MESA Research Institute, University of Twente, The Netherlands, November, 21-22 1994) (Boston: Kluwer)

Webster J R and Mastrangelo C H 1997 Large volume integrated capillary electrophoresis stage fabricated using micromachining of plastics on silicon substrates Int. Conf. on Solid-State Sensors Actuators: Transducers '97 p 503
Webster J R, Burns M, Burke D and Mastrangelo C 1998 An inexpensive plastics technology for microfabricated capillary electrophoresis chips Conf. Proc. MicroTAS'98 (October 12-16, 1998)

Wilkes J A and Churchill S W 1966 The finite difference computation of natural convection in a rectangular enclosure AIChE J. 12161

Yarnold G D 1938 The motion of a mercury index in a capillary tube Proc. R. Soc. $\mathbf{5 0} 540$ 